\title{
Identifying Dynamic Discrete Decision Processes
}

\author{
Thierry Magnac*, INRA-LEA and CREST \\ David Thesmar, INSEE and CREST
}

First version: March 1998

This version: January 2001

\begin{abstract}
In this paper, we analyse the non parametric identification of dynamic discrete choice models using short-panel data. Our identification methodology is based on the ideas explored in the seminal paper of Hotz and Miller (1993) that Bellman equations can be interpreted as moment conditions. We derive the exact degree of underidentification of these models both in the case where random shocks on preferences are independent over time and in a case with correlated fixed effects. We investigate the necessity and power of various identifying restrictions.
\end{abstract}

Keywords: Dynamic discrete choice processes, identification.

JEL classification: C25, D91, C14

${ }^{*}$ Corresponding author: Thierry Magnac, INRA-LEA, 48, Boulevard Jourdan, 75014 Paris, France. Email: Thierry.Magnac@ens.fr 


\section{Introduction ${ }^{1}$}

Over the last decade, the number of econometric applications using structural models of dynamic discrete choice has been growing quickly. These models allow economists to simulate the consequences of economic policies affecting education, labor market transitions, retirement decisions or fertility choices (see for example Keane and Wolpin, 1996, Eckstein and Wolpin, 1989). Little is known however about the identification of these models except that they are generically not identified (Rust, 1994).

In this paper, we analyse the non parametric identification of dynamic discrete choice models. Our methodology is based on the insight of Hotz and Miller (1993) that Bellman equations can be interpreted as moment conditions. We consider cases with and without unobserved heterogeneity. Not only do we show that these models are not identified (Rust, 1994), we are also able to determine their exact degree of underidentification. We begin with the case without correlated unobserved heterogeneity. Using Bellman equations as moment conditions, we show that utility functions in each alternative cannot be (non parametrically) identified as long as the following structural parameters are not set: the distribution function of unobserved preference shocks, the discount rate and the current and future preferences in one (reference) alternative. We also investigate how exclusion or parametric restrictions can provide identifying restrictions. As the identification proof is constructive, a simple method of moment estimator can be derived and overidentifying restrictions can be tested. Provided that one is willing to make stronger identifying assumptions, dynamic discrete choice modelling is thus little different from the continuous case. Bellman equations can be used to recover deep structural parameters as Euler equations are. We continue by exploring a case where the unobserved component of preferences is correlated over time. Even if the functional degree of underidentification of this model is larger, we present reason-

\footnotetext{
${ }^{1}$ We would like to acknowledge helpful comments by three anonymous referees, helpful discussions with G. van den Berg, J.M. Dufour, A. Kirman, F. Kramarz, V. Patilea and participants in seminars at CREST, University College, LSE, Louvain-la-Neuve, Montreal, Madrid, Toulouse, Cergy-Pontoise, Orléans, Marseille and in conferences, ESEM, Berlin, JAE, Rotterdam, SED, Alghero and ESRC, Bristol. The usual disclaimer applies.
} 
able identifying assumptions that lead to the same identification results as without unobserved heterogeneity. The same methodology using moment conditions is applied.

This paper expands upon the work in Rust (1994), where the generic non identification result is stated. We use a slightly different model. In our case, agents' preferences have unobservable and possibly persistent components. The constructive aspect of our proof allows us to interpret Rust's underidentification result and to propose identifying restrictions. On the technical side, the insights for our identification strategy are borrowed from the works of Hotz and Miller (1993), Hotz et al. (1994) and Altug and Miller (1998). We express Bellman equations as moment conditions in a different way than they do. This allows us to dispense with the existence of an absorbing state or simulation procedures, and to investigate identification, an issue not previously addressed by these authors. Furthermore, correlated unobserved heterogeneity is only considered in some applications using maximum likelihood methods and backward induction (Keane and Wolpin, 1996, Eckstein and Wolpin, 1999). These procedures are computer intensive and their degree of complexity is large (Rust, 1996). The way these models are identified is not clear because it is more difficult to discuss identification in a maximum likelihood framework. Our paper also bears some resemblance to Cameron and Heckman (1998), who develop identification results in a dynamic setting with correlated additive fixed effects. In their framework however, agents are assumed to be myopic and semiparametric preferences are assumed.

Section 2 establishes the theoretical model without correlated individual effects. Section 3 provides the main proposition about identification and provides estimable moment conditions. In section 4, the identifying power of various restrictions is analyzed. Section 5 provides the extension to a case where individual effects are correlated. Section 6 concludes the paper.

\section{The theoretical set-up}

This section presents the general class of dynamic discrete decision models that we focus on in this paper. We first define notations, state assumptions on preferences 
and expectations and derive Bellman equations that are the structural restrictions in a dynamic and stochastic framework. Second, we define identification.

\subsection{The structural model.}

Consider a decision maker whose intertemporal utility is additively time separable and whose instantaneous preferences are defined over a discrete set of alternatives $i \in I=\{1, ., K\}$. Within-period utility functions are not restricted, even by general constraints such as monotonicity or concavity restrictions (Matzkin, 1994).

State variables that the decision maker considers are denoted $h$. Some of them, called $x$, can be observed by the econometrician ${ }^{2}$. Others are random preference shocks $\varepsilon=\left(\varepsilon_{1}, . ., \varepsilon_{K}\right)$ in each alternative $i$. Let $h=(x, \varepsilon)$. Within a period, the sequence of events is the following. At the beginning of the period, both $x$ and $\varepsilon$ are revealed to the agent who then chooses an alternative $d=i$, and gets the utility value $u_{i}\left(x, \varepsilon_{i}\right)$. Next period state variables $h^{\prime}=\left(x^{\prime}, \varepsilon^{\prime}\right)$ are then drawn conditional on $h=(x, \varepsilon)$ and the observed decision, $d=i$. In other words, $h$ is assumed to follow a Markov process of order one. ${ }^{3}$ We also restrict the way preferences, $u_{i}$, depend on $\varepsilon_{i}$ :

Assumption AS (Additive Separability) Instantaneous utility functions are given by:

$$
\forall i \in I ; u_{i}(x, \tilde{\varepsilon})=u_{i}^{*}(x)+\varepsilon_{i}
$$

where:

i. $\tilde{\varepsilon}=\left(\varepsilon_{1}, ., \varepsilon_{K}\right)$ are mean-independent of $x$ :

$$
E\left(\varepsilon_{i} \mid x\right)=0 .
$$

ii. Its cumulative distribution function, denoted $G$, is absolutely continuous with respect to the Lebesgue measure in $\mathbb{R}^{K}$.

\footnotetext{
${ }^{2}$ Time is assumed to be one of these observed state variables in order to simplify the presentation.

${ }^{3}$ The only loss of generality could be that random shocks are Markov of higher order. See below assumption (CI) to rule out this case.
} 
Along with assumption CI stated below, assumption $\operatorname{AS}(i)$ ensures that

$$
E\left(u_{i}(x, \widetilde{\varepsilon}) \mid x\right)=u_{i}(x, \tilde{\varepsilon})-\varepsilon_{i}
$$

can be interpreted as a structural parameter, that is to say, as the part of the utility function that depends on the observable state variables. As for $\mathrm{AS}(i i)$, some authors (Hotz and Miller, 1993, for instance) consider that the distribution function $G$ depends on $x$. This implies, for example, that the variance of random preferences depends on $x$. In order to reduce expository noise as much as possible, we omit this dependence because our results are affected by this assumption in an obvious way that we will briefly discuss. Finally, absolute continuity of $G$ must hold if we want the economic model to have a unique optimal solution for all $\tilde{\varepsilon}$ (except in a set of Lebesgue-measure zero). This result has been established in the static discrete case by Koning and Ridder (1996). ${ }^{4}$

Next, we need to make assumptions on how agents' beliefs (noted $\mu$ ) are related to real life transition probabilities of $h=(x, \varepsilon)$. To focus on the identifying power of intertemporal optimization, we assume that expectations are perfect (see Buchinsky and Leslie (1997) for the implications of alternative expectation formation processes):

Assumption PE Agents have perfect expectations on the law of motion from $h=(x, \widetilde{\varepsilon})$ and $d=i$ to $h=\left(x^{\prime}, \widetilde{\varepsilon}^{\prime}\right)$. That is, for all $h, d=i, h^{\prime}$ :

$$
\mu\left(h^{\prime} \mid h, d=i\right)=\operatorname{Pr}\left(h^{\prime} \mid h, d=i\right) .
$$

As time is included in the observable state variable $x, \mathrm{PE}$ implicitly means that macro shocks are known ex ante by agents. It is quite a strong assumption though not surprising. In the Euler equation framework, it is indeed the basis of the Chamberlain (1984) critique. Cross-section identifying restrictions given by Euler equations are valid only if macro shocks and the way agents perceive them are restricted. As we are looking for conditions for cross-section identification in Discrete Decison Process (DDP) models, it is natural to begin with the case where macro shocks are known.

\footnotetext{
${ }^{4}$ Note that the proof of non identification given in Rust (1994) does not apply here since he assumes that $\tilde{\varepsilon}=0$.
} 
In this set-up, perfect expectations cannot be derived from a learning process based on observations (Manski, 1993). The optimal decision is a deterministic function of the current state variables $H=(x, \widetilde{\varepsilon})$. Out of equilibrium events have, therefore, zero probability. Transition probabilities conditional on out of equilibrium events cannot be observed, and future payoffs of decisions cannot be learned. In such a set-up, agents' beliefs have to be exogenously coordinated on the true transition probabilities.

It is however possible to reconcile perfect expectations and learning in this set-up provided we make the following additional assumption :

Assumption CI (Conditional Independence Assumption) Random preference shocks at two periods $\tilde{\varepsilon}^{\prime}$ and $\widetilde{\varepsilon}$ are independent conditional on $(x, d=i)$.

Condition CI ensures that all unobservable state variables are independant. The econometrician can observe all state variables that display some persistence. In the last section of this paper, we relax assumption CI. Its usual interpretation is that it rules out unobserved heterogeneity (Rust, 1994), and dynamic selection biases as in Cameron and Heckman (1998).

Provided CI holds, the transition matrix of the process governing $(x, \varepsilon)$ is written as:

$$
\operatorname{Pr}\left(h^{\prime} \mid h, d\right)=\operatorname{Pr}\left(x^{\prime}, \tilde{\varepsilon}^{\prime} \mid x, d\right)
$$

so that conditional probabilities can be inferred by the agent from observing other selfs (Manski, 1993) and future payoffs of current decisions can be computed.

As a consequence of assumptions AS, CI and PE, Bellman equations relating current value functions in each alternative and future value functions are:

$$
v_{i}(x, \tilde{\varepsilon})=u_{i}(x)+\varepsilon_{i}+\beta E\left(\max _{j} v_{j}\left(x^{\prime}, \tilde{\varepsilon}^{\prime}\right) \mid x, d=i\right)
$$

where $\beta$ is the discount factor. From assumption PE, the agent integrates with respect to the true distribution function of future state variables $x^{\prime}, \tilde{\varepsilon}^{\prime}$, conditional on $d=i$ and $x$. From (2.1), value functions can be decomposed as:

$$
v_{i}(x, \tilde{\varepsilon})=v_{i}^{*}(x)+\varepsilon_{i}
$$


and the deterministic part, $v_{i}^{*}(x)$ is the solution to:

$$
v_{i}^{*}(x)=u_{i}^{*}(x)+\beta E\left(\max _{j}\left(v_{j}^{*}\left(x^{\prime}\right)+\varepsilon_{j}^{\prime}\right) \mid x, d=i\right)
$$

that, in the following, we shall improperly call Bellman equations.

\subsection{Definition of identification}

We define and prove results in the two-period case although the argument can easily be extended to any finite number of periods. To fix ideas, , in the following, we consider the discrete and finite case.

\section{Assumption DS (Discrete Support Assumption)}

The support of first-period state variables $x$ (resp. second-period $x^{\prime}$ ) is $X$ (resp. $\left.X^{\prime}\right)$. The joint support $\tilde{X}=X \cup X^{\prime}$ is discrete and finite i.e.:

$$
\tilde{X}=\left\{x_{1}, ., x_{\# X}\right\}
$$

Under DS, the identification of the structural form boils down to the identification of the values of the utility function and the transition probabilities at a finite number of points only. It is, however, easy to adapt the proofs of identification that follow, to the case, for instance, where the set $\tilde{X}$ is a compact set and the functions to be identified are continuously differentiable. ${ }^{5}$

The data provide us with the following function:

$$
\forall\left(d, d^{\prime}\right) \in I^{2}, \forall\left(x, x^{\prime}\right) \in X \times X^{\prime}, \operatorname{Pr}\left(d^{\prime}, x^{\prime}, d \mid x\right)=\operatorname{Pr}\left(d^{\prime} \mid x^{\prime}\right) . \operatorname{Pr}\left(x^{\prime} \mid x, d\right) . \operatorname{Pr}(d \mid x) .
$$

First, it should be noted that structural transition probabilities, $\operatorname{Pr}\left(x^{\prime} \mid x, d\right)$, combined with assumption PE on agent's expectations, directly permits identification of agents' beliefs about the Markov process from $x, d$ to $x^{\prime}$. To simplify the notation, we now remove this structural object from the list of parameters to be identified. Other deep structural parameters affect choice probabilities $\operatorname{Pr}(d \mid \tilde{x})$, $\tilde{x} \in \tilde{X}$. These parameters are:

\footnotetext{
${ }^{5}$ It seems, however, natural to consider the discrete case first, since decisions take their values in a discrete and finite set $\{1, ., K\}$. Thus, histories inherently have discrete components. Furthermore, DS allows us to abstract from the identifying power of continuity and differentiability restrictions.
} 
- Current behavior: the current period utility functions, $\left(u_{1}^{*}(x), . ., u_{K}^{*}(x)\right)$, for all $x \in X$.

- Future behavior: second period value functions,: $\left(v_{1}^{*}\left(x^{\prime}\right), . ., v_{K}^{*}\left(x^{\prime}\right)\right)$ for all $x^{\prime} \in X^{\prime}$ and the discount rate $\beta$.

- Expectations: the subjective belief over the Markov process of preference shocks, $\mu\left(\widetilde{\varepsilon}^{\prime} \mid x^{\prime}\right)$ which is equal to $G$ by assumption PE.

Second period value functions are structural objects here because we focus on short panel identification. In a model of longer time span, they would be interpreted as reduced forms of future preferences. In our short panel context, the structure of the model is therefore defined by parameters:

$$
b=\left\{u_{1}^{*}(X), . ., u_{K}^{*}(X), v_{1}^{*}\left(X^{\prime}\right), . ., v_{K}^{*}\left(X^{\prime}\right), G, \beta\right\}
$$

where $f(X)$ is a short-cut for $\{f(x) ; \forall x \in X\}$.

A structure $b$, combined with Bellman equations (2.2) permits computation of value functions $v_{i}^{*}(\tilde{x} ; b)$ for all $\tilde{x} \in \tilde{X}$ and $i \in I$. The agent is rational and chooses alternative $i$ if and only if $i$ yields the maximum value. Let $p_{i}(\tilde{x} ; b)$ be the probability that the agent chooses $d=i$ given the structure $b$ and observed state variable $\tilde{x}$ :

$$
\forall(\tilde{x}, i) \in \tilde{X} \times I ; p_{i}(\tilde{x} ; b)=\operatorname{Pr}\left(v_{i}^{*}(\tilde{x} ; b)+\varepsilon_{i}=\max _{j}\left(v_{j}^{*}(\tilde{x} ; b)+\varepsilon_{j}\right) \mid \tilde{x}, b\right)
$$

Predicted behavior $p_{i}(\tilde{x} ; b)$ is called the reduced form of the structural model. If the true structure is $b_{0}$, reduced form choice probabilities must equal those observed :

$$
\forall(\tilde{x}, d), \underbrace{\operatorname{Pr}(d \mid x)}_{\text {Data }}=\underbrace{p_{d}\left(\tilde{x} ; b_{0}\right)}_{\text {Model }}
$$

To define identification, we start with the notion of observational equivalence between two structures $b$ and $b^{\prime}$ if they lead to the same reduced forms.

Definition 2.1. (Observational equivalence) Let $B$ be the set of structures $b$, and $\stackrel{o}{\Longleftrightarrow}$ denote "observational equivalence". Let two structures $\left(b, b^{\prime}\right) \in B^{2}$ :

$$
b \stackrel{o}{\Longleftrightarrow} b^{\prime} \text { if and only if } \forall(\tilde{x}, d), p_{d}\left(\tilde{x} ; b^{\prime}\right)=p_{d}(\tilde{x} ; b) \text {. }
$$


In other words, the model does not make predictions that differ enough to select one of the two structures. The model is thus said to be identified by the data if it is always possible to discriminate among two structures:

Definition 2.2. (Identification) The model is identified if and only if:

$$
\forall b, b^{\prime} \in B^{2},\left(b \stackrel{o}{\Longleftrightarrow} b^{\prime}\right) \Rightarrow\left(b=b^{\prime}\right)
$$

\section{Identification Results and Estimation}

We are now in a position to state the identification result.

\subsection{Identification}

For any $\tilde{x} \in \tilde{X}$, denote the vector of choice probabilities $\tilde{p}(\tilde{x})=\left(p_{1}(\tilde{x}), ., p_{K}(\tilde{x})\right)$ and the vector of value functions $\tilde{v}^{*}(\tilde{x})=\left(v_{1}^{*}(\tilde{x}), ., v_{K}^{*}(\tilde{x})\right)$. Given $G$, equation (2.3) maps $\tilde{v}^{*}(\tilde{x})$ into $\tilde{p}(\tilde{x})$. Hotz and Miller (1993) show that these equations can be inverted as:

$$
\forall(\tilde{x}, i) \in \tilde{X} \times I ; v_{i}^{*}(\tilde{x})=v_{K}^{*}(\tilde{x})+q_{i}(\tilde{p}(\tilde{x}) ; G)
$$

where $q_{i}($.$) are functions mapping the simplex set in \mathbb{R}^{K}$ onto $\mathbb{R}$. These functions are derived from $G$ and by definition, $q_{K}()=$.0 . Furthermore, $q=\left(q_{1}, \ldots, q_{K}\right)$.

For given $G$ and $v_{K}^{*}\left(X^{\prime}\right)$, then it is always possible to recover $v_{i}^{*}\left(X^{\prime}\right), i<K$ from choice probabilities $\tilde{p}\left(X^{\prime}\right)$. From (3.1), we get:

$$
\left(v_{1}^{*}\left(X^{\prime}\right), ., v_{K-1}^{*}\left(X^{\prime}\right)\right)=\varphi\left(\tilde{p}\left(X^{\prime}\right) ; v_{K}^{*}\left(X^{\prime}\right)\right)
$$

If $z=\left(z_{1}, ., z_{K}\right)$, we also define function $R: \mathbb{R}^{K} \rightarrow \mathbb{R}$ such that:

$$
R(z ; G)=E_{G} \max _{i \in I}\left(z_{i}+\varepsilon_{i}-\varepsilon_{K}\right)
$$

Using this definition and (3.1), we rewrite Bellman equations (2.2):

Lemma 3.1. Bellman equations (2.2) can be expressed for any $i \in\{1, ., K-1\}$ as:

$$
\begin{gathered}
u_{i}^{*}(x)=u_{K}^{*}(x)-\beta\left(E\left(v_{K}^{*}\left(x^{\prime}\right) \mid x, d=i\right)-E\left(v_{K}^{*}\left(x^{\prime}\right) \mid x, d=K\right)\right) \\
+q_{i}(\tilde{p}(x) ; G)-\beta\left(E\left(R\left(q\left(\tilde{p}\left(x^{\prime}\right) ; G\right) ; G\right) \mid x, d=i\right)-E\left(\left(R\left(q\left(\tilde{p}\left(x^{\prime}\right) ; G\right) ; G\right) \mid x, d=K\right)\right) .\right.
\end{gathered}
$$


Proof. See appendix A.

This lemma proves that Bellman equations can be written in terms of (1) utility functions $\left(u_{1}^{*}(X), \ldots, u_{K-1}^{*}(X)\right),(2)$ the other deep structural parameters $\beta, G, u_{K}^{*}(X), v_{K}^{*}\left(X^{\prime}\right)$ and $(3)$ the choice probabilities $\tilde{p}(\tilde{X})=\left(p_{1}(\tilde{X}), \ldots, p_{K}(\tilde{X})\right)$, that are observed from the data. The last Bellman equation in (2.2) defines $v_{K}(X)$ as a function of $b$ (see appendix $\mathrm{A}$ ) and is therefore not informative as $v_{K}$ is not a structural parameter. Lemma 3.1 therefore provides a mapping between the data and the utility functions in the first $K-1$ alternatives at a given value of the other structural parameters. More formally:

$$
\underbrace{\left(u_{1}^{*}(X), \ldots, u_{K-1}^{*}(X)\right)}_{\text {utility function }}=\phi(\underbrace{\tilde{p}(X), \pi}_{\text {data }} ; \underbrace{\beta, G, u_{K}^{*}(X), v_{K}^{*}\left(X^{\prime}\right)}_{\text {Structure }})
$$

where $\pi\left(=\operatorname{Pr}\left(X^{\prime} \mid X, I\right)\right)$ are the structural transition probabilities defined in the previous section and over which the expectation operator in (3.3) is taken. Identification can now be characterized:

Proposition 3.2. Let $C=\left\{c \mid c=\left(\beta, G, u_{K}^{*}(X), v_{K}^{*}\left(X^{\prime}\right)\right)\right\}$ be the set of possible discount factors, random preference shocks d.f. and preferences in the reference alternative.

1. Fix $c \in C$. There exists only one vector $\left(u_{1}^{*}(X), \ldots, u_{K-1}^{*}(X)\right)$ compatible with the data $\widetilde{p}(\tilde{X})$.

2. Dynamic discrete choice models are not identified. For two different elements of $C$, it is always possible to find other structural parameters and, in particular, utility functions, that are compatible with the data:

$$
\forall c, c^{\prime} \in C \times C,\{\phi(. ; c), \varphi(. ; c), c\} \stackrel{O}{\Leftrightarrow}\left\{\phi\left(. ; c^{\prime}\right), \varphi\left(. ; c^{\prime}\right), c^{\prime}\right\}
$$

where $\phi$ and $\varphi$ are defined in (3.2) and (3.4).

Proof. See appendix B.

This proposition shows that utility functions are far from identified. The degree of underidentification is large since it consists in $\beta, G$ and preferences in the reference alternative $\left(u_{K}^{*}(X), v_{K}^{*}\left(X^{\prime}\right)\right)$. It would be even larger if $G$ or $\beta$ were to depend on $x$. 
An implication of proposition 3.2 is well known in the literature. Manski (1993, p129) proves that the discount rate is not identified because any DDP model (i.e. $\beta \neq 0$ ) is observationally equivalent to a static model (i.e. $\beta=0$ ). To some extent, terminal conditions matter too, since future preferences $v_{K}^{*}\left(X^{\prime}\right)$ in the reference alternative are necessary to identify current preferences. Below we discuss more extensively the identifying power of terminal date restrictions.

Yet, the arbitrariness of future preferences in the reference alternative is " less" important than the arbitrariness of $\beta$ and $G$. Assume now that $v_{K}^{*}\left(X^{\prime}\right)$ is unknown. An interesting structural object can still be identified. Rearranging equations from lemma 3.1, we see that $\forall i \in\{1, ., K-1\}$ :

$$
\begin{aligned}
& q_{i}(\tilde{p}(x) ; G)-\beta\left(E\left(R\left(q\left(\tilde{p}\left(x^{\prime}\right) ; G\right) ; G\right) \mid x, d=i\right)-E\left(\left(R\left(q\left(\tilde{p}\left(x^{\prime}\right) ; G\right) ; G\right) \mid x, d=K\right)\right)\right. \\
= & {\left[u_{i}^{*}(x)+\beta E\left(v_{K}^{*}\left(x^{\prime}\right) \mid x, d=i\right)\right]-\left[u_{K}^{*}(x)+\beta E\left(v_{K}^{*}\left(x^{\prime}\right) \mid x, d=K\right)\right] } \\
\equiv & U_{i}(x)
\end{aligned}
$$

Given $\beta$ and $G$, the LHS can be identified. The RHS, $U_{i}(x)$, measures the difference between the expected values of two sequences of choices: first, choose $i$ now, $K$ tomorrow, and behave optimally afterwards; second, choose $K$ now and tomorrow and behave optimally afterwards. In the following, we call $U_{i}($.$) the current value$ function of $i$. It is identified even if future preferences are not restricted.

Corollary 3.3. Fix $\beta$ and $G$. Then, $\forall i \in\{1, ., K-1\}, U_{i}(X)$ can be uniquely recovered from the data.

This corollary clarifies the difference between results that can be obtained in the static case $(\beta=0)$ and in the dynamic case $(\beta \neq 0)$. In the static model, equation (3.5) means that the difference between the levels of utility functions, $u_{i}^{*}()-.u_{K}^{*}($.$) ,$ is equal to $U_{i}($.$) . It is therefore identified. In the dynamic case however, differences$ between levels cease to be identified unless the continuation value function $v_{K}\left(X^{\prime}\right)$ of the reference alternative is given.

In the next section, we examine other identifying restrictions after using lemma 3.1 to infer an estimation strategy of the current value function $U_{i}($.$) .$ 


\subsection{Estimation strategy}

The construction of our identification proof suggests a method-of-moment estimation strategy in two steps. Bellman equations from lemma 3.1 are analogous to Euler equations in the continuous choice case. As in Hotz and Miller (1993), the first step consists in estimating choice probabilities $\tilde{p}(\tilde{X})$. Denote $\hat{p}_{n}(\tilde{X})$ such an estimate, which under assumption DS, is the shares of chosen alternatives in the subsamples $x=x_{s}$.

The second step differs. Hotz and Miller (1993) and Hotz et al. (1994) express expected continuation values as functions of all future utilities which are parametric functions. Hotz and Miller (1993) show that, if a state is absorbing, expected continuation values are functions of both next-period utilities and choice probabilities. In lemma 3.1, we proved that expected continuation values are functions of choice probabilities only. We use the resulting equations as the only moment conditions of the problem.

Suppose first that $\tilde{p}(\tilde{X})$ is known. Given arbitrary values for $\beta$ and $G$, for every observation such that $d=i$, we can compute :

$$
Y_{i}=q_{i}(\tilde{p}(x))-\beta R\left(q\left(\tilde{p}\left(x^{\prime}\right)\right)\right.
$$

Remark that (3.5) implies that:

$$
U_{i}(x)=E\left(Y_{i} \mid x, d=i\right)-E\left(Y_{K} \mid x, d=K\right)
$$

We can therefore estimate $U_{i}(X)$ using subsamples of individuals having chosen $d=1, ., K$ since:

$$
Y_{i}=E\left(Y_{i} \mid x, d=i\right)+\eta_{i}
$$

where $E\left(\eta_{i} \mid x, d=i\right)=0$. This corresponds to the Euler equation but in a discrete case. Moreover, subsamples are independent and the estimation of $E\left(Y_{i} \mid x, d=i\right)$ and $E\left(Y_{K} \mid x, d=K\right)$ are independent.

In fact, variables $Y_{i}$ cannot be computed from the data since $\tilde{p}(\tilde{X})$ is unknown. What can be computed from the data is the quantity:

$$
\hat{Y}_{\text {in }}=q_{i}\left(\hat{p}_{n}(x)\right)-\beta R\left(q\left(\hat{p}_{n}\left(x^{\prime}\right)\right) .\right.
$$


The empirical model can therefore be rewritten as:

$$
\hat{Y}_{i n}=E\left(Y_{i} \mid x, d=i\right)+\eta_{i}+\hat{w}_{i n}
$$

where $\hat{w}_{i n}=\hat{Y}_{n i}-Y_{n i}$ is the sampling error of the first step. Results concerning two-step estimates should therefore be used (Newey and McFadden, 1994). The different equations are not independent any longer since the presence of $\hat{p}_{n}(x)$ in all equations induces cross-equation correlations.

\section{Identifying Power of Various Restrictions}

In this section, we evaluate the identifying power and testability of restrictions that are used in the literature on dynamic discrete decision processes. We start with showing that some usual assumptions like the presence of absorbing states or terminal dates, do not have additional identifying power. We continue by examining the identifying power given by exclusion restrictions or parametric restrictions.

\subsection{Absorbing states and terminal dates}

It is often assumed that one state is absorbing (e.g. Hotz and Miller, 1993). Let $K$ (the reference alternative) be the absorbing state. The future choice set is restricted to $\{K\}$ when alternative $K$ is chosen in the current period. The $K$ th Bellman equation is then written :

$$
v_{K}^{*}(x)=u_{K}^{*}(x)+\beta E\left(v_{K}^{*}\left(x^{\prime}\right) \mid x, d=K\right)
$$

which implies that $E\left(R\left(q\left(\tilde{p}\left(x^{\prime}\right) ; G\right) ; G\right) \mid x, d=K\right)=0$. This restriction is not informative about the structure $b$. It is indeed testable from the data.

It is often assumed in the literature on maximum likelihood estimation of dynamic discrete choices that some date $T$ is a terminal date. Assume that value functions at $T$ in some alternatives $i$ are known and equal to $v_{i}^{(T) *}($.$) (Keane and$ Wolpin, 1996, Belzil and Hansen, 1997). If choice probabilities can be recovered from the data at date $T$, these restrictions are testable unless they only concern the value function of the reference alternative $v_{i}^{(T) *}($.$) . Yet, this last restriction on$ $v_{i}^{(T) *}($.$) would fall far short of from solving the underidentification problem. From$ 
proposition 3.2, in order to identify utility functions, assumptions on preferences in the reference alternative have to be made at every period.

\subsection{Exclusion restrictions or parametric restrictions}

To identify $\beta$, restrictions can be imposed by excluding one variable from current value functions as we now show. An exclusion restriction takes the form of :

$$
R: \exists\left(x_{1}, x_{2}\right) \in X^{2}, \exists i \in I \text { such that: } x_{1} \neq x_{2} \text { and } U_{i}\left(x_{1}\right)=U_{i}\left(x_{2}\right) .
$$

Then:

Proposition 4.1. Fix $G$. Add the exclusion restriction $R$ to the set of structural restrictions of the model. Provided a rank condition given in the proof is satisfied, $U_{i}(X)$ and $\beta$ are just or over identified.

Proof. Use (3.5) for $\left(i, x_{1}\right)$ and $\left(i, x_{2}\right)$ and subtract. As $U_{i}\left(x_{1}\right)=U_{i}\left(x_{2}\right)$, it yields a linear equation in $\beta$ where $\beta$ is the only unknown. The coefficient of $\beta$ can be determined provided the following coefficient:

$$
\begin{array}{r|l}
{\left[E \left(R \left(q\left(\tilde{p}\left(x^{\prime}\right)\right)\right.\right.\right.} & \left.\mid \quad H=x_{1}, d=i\right)-E\left(R\left(q\left(\tilde{p}\left(x^{\prime}\right)\right) \mid H=x_{1}, d=K\right)\right] \\
-\left[E \left(R \left(q\left(\tilde{p}\left(x^{\prime}\right)\right)\right.\right.\right. & \left.\mid \quad H=x_{2}, d=i\right)-E\left(R\left(q\left(\tilde{p}\left(x^{\prime}\right)\right) \mid H=x_{2}, d=K\right)\right]
\end{array}
$$

is different from 0 for at least two histories $x_{1}$ and $x_{2}$. If there are more than one exclusion restrictions and corresponding rank conditions, $\beta$ is overidentified.

Stationarity of current value functions is a particular case of exclusion restrictions when there are more than two periods. Assume that there are three periods and apply corollary 3.3 to show that $U_{i}(X)$ and $U_{i}\left(X^{\prime}\right)$ are identified. Stationarity implies restriction $R$, since there exist $x_{s} \in X, x_{s^{\prime}} \in X^{\prime}$ and $i$ such that $U_{i}\left(x_{s}\right)=U_{i}\left(x_{s^{\prime}}\right)$.

Finally, parametric restrictions on utility functions are very often used (Hotz and Miller, 1993, Keane and Wolpin, 1996) ${ }^{6}$. As our framework consists of using equations (3.5) as moment conditions, these restrictions can readily be implemented and tested ${ }^{7}$.

\footnotetext{
${ }^{6}$ In our case where the support is discrete, a parametric specification means that the utility functions are functions of a number of parameters which is strictly less than the number of support points of $S$ times $K-1$. They are therefore equivalent to exclusion restrictions.

${ }^{7}$ Parametric restrictions cannot be used to identify d.f. $G$ because histories have discrete support (see Manski, 1988).
} 


\section{Correlated fixed effects}

In this section, we consider that preferences are affected by correlated fixed effects. The reader might not be surprised that the underidentification result carries over to this case. We, however, present identifying restrictions that are sufficient to identify structural parameters and the distribution of unobserved heterogeneity.

For internal consistency reasons, perfect expectations (assumption PE) need to be grounded in observation. Therefore, agents need to have beliefs about the distribution of random shocks $\widetilde{\varepsilon}$ and the transition probabilities conditional on their own fixed effect (Manski, 1992). An example of such beliefs is that these probability distributions do not depend on the fixed effect (assumptions CI and AS). When observation of the types of the other agents is not possible, agents cannot refute these latter beliefs against the general alternative that shocks $\varepsilon$ are correlated. Therefore, under the assumption that the agents believe in the absence of permanent heterogeneity, AS and CI become untestable.

When agents can observe the fixed effects of other agents, they can infer probability distributions conditional on types. They can therefore refute AS and CI. In this context, knowledge of their own types allows agents to form more accurate expectations and gives them an informational advantage over econometricians.

\subsection{The general framework}

The type of the agent is described by a one-dimensional state variable $\theta$, constant over time. It is observed by all other agents but not by the econometrician. As the state space is modified and described by $h=(x, \theta, \widetilde{\varepsilon})$. Assumptions of additive separability, conditional independence and perfect expectations, are therefore amended to make them conditional on observed state variables $x$ and the type of the agent. In other words, we replace $x$ in $\mathrm{AS}, \mathrm{CI}$ and $\mathrm{PE}$ by $(x, \theta)$. It is a world where there are as many Bellman equations as unobserved types and equation (2.2) can be rewritten as:

$$
v_{i}^{*}(x, \theta)=u_{i}^{*}(x, \theta)+\beta E\left(\max _{j}\left(v_{j}^{*}\left(x^{\prime}, \theta\right)+\varepsilon_{j}^{\prime}\right) \mid d=i, x, \theta\right)
$$


The difficulty with respect to the homogenous case arises from the presence of dynamic selection or endogeneity biases (Cameron and Heckman, 1998, Taber, 2000) because one state-variable, $\theta$, remains unobserved by the econometrician. Observed histories and random shocks are no longer independent. The additivity in $\theta$ of $u_{i}^{*}(x, \theta)$ can be used to identify structural parameters if the decision maker is myopic as shown by Cameron and Heckman. In the general case however, the value function $v_{i}^{*}(x, \theta)$, given by equation (5.1), is not linear in $\theta$ even if $u_{i}^{*}(x, \theta)$ is linear in $\theta$ because of the convexity of the max-operator. Cameron and Heckman's proof should therefore be extended to account for non myopic behavior.

\subsection{The degree of underidentification}

For simplicity to highlight the main issues, we will, from now on, restrict the model and consider only two alternatives. Preferences in the reference alternative $\left(u_{2}^{*}(X, \theta), v_{2}^{*}\left(X^{\prime}, \theta\right)\right)$ are assumed to be equal to zero and state 2 is absorbing. Instantaneous utility in alternative 1 is given by:

$$
u_{1}(h)=u_{1}^{*}(x, \theta)+\varepsilon_{1} .
$$

Moreover, suppose that $\theta$ can only take ${ }^{8}$ two values 0 and 1 , and that, at the initial date, the distribution $r(\theta \mid x)=r(\theta)$ is independent of $x$. We first focus on the case where the heterogeneity, $\theta$, only affects preferences, and not the transition process of $h=(x, \varepsilon)$ :

$$
\operatorname{Pr}\left(x^{\prime} \mid x, d, \theta\right)=\operatorname{Pr}\left(x^{\prime} \mid x, d\right) \text { and } G(\varepsilon \mid \theta)=G(\varepsilon)
$$

Even under these stringent assumptions, there are dynamic selection biases since tastes are correlated over time. The structural equations at two dates 0 and 1 are summarized in the following lemma:

Lemma 5.1. Let $G$ be a distribution function, and $q$ and $R$ the associated two mappings as defined in section 3.1. Denote $p(x, \theta)$ as the probability of choosing

\footnotetext{
${ }^{8}$ It does not seem that the extension to any given number of types changes the results that follow. The restriction on the values of $\theta$ is of limited impact since the location and scale of utility functions are not identified because $G$ is not identified.
} 
alternative 1 conditional on type $\theta$, and $s(x)$ as the unconditionnal (observed) probability. Then:

$$
\left\{\begin{aligned}
\theta=0,1 \quad u_{1}^{*}(x, \theta) & =q(p(x, \theta) ; G)-\beta E\left(R\left(q\left(p\left(x^{\prime}, \theta\right) ; G\right) ; G\right) \mid d=1, x\right) \\
s(x) & =p(x, 1) r(1)+p(x, 0) r(0) \\
s\left(x^{\prime}\right) & =p\left(x^{\prime}, 1\right) \frac{p(x, 1) r(1)}{s(x)}+p\left(x^{\prime}, 0\right) \frac{p(x, 0) r(0)}{s(x)} .
\end{aligned}\right.
$$

Proof. See Appendix C.

The first line comes from the Bellman equations, as in lemma 3.1. The difference now is that there are two such equations for alternative 1, because there are two unobserved types. The following lines relate the choice probabilities $s(x)$, identifiable as a population parameter, to the unknown type dependent choice probabilities. These Bayes equations provide additional structural restrictions, that have to be satisfied. The difference in weights between periods of the two types in the population illustrates the presence of dynamic selection.

Given these moment conditions, the degree of underidentification is given by:

Proposition 5.2. Fix $G$ and $\beta$. Let $p\left(x^{\prime}, 1\right)$ and $p(x, 1)$ be two arbitrary choice probabilities taking their values between 0 and 1 such that $\exists \alpha_{0}>0$ and:

$$
\begin{gathered}
\min \left(\frac{s(x)}{p(x, 1)}, \frac{1-s(x)}{1-p(x, 1)}\right) \geq \alpha_{0} \\
\frac{s(x)}{p(x, 1)} \min \left(\frac{s\left(x^{\prime}\right)}{p\left(x^{\prime}, 1\right)}, \frac{1-s\left(x^{\prime}\right)}{1-p\left(x^{\prime}, 1\right)}\right) \geq \alpha_{0} .
\end{gathered}
$$

Consider an arbitrary value $0<r(1) \leq \alpha_{0}$. Then $u_{1}^{*}(x, \theta)$ is just identified.

Proof. Consider the second equation in (5.3). As $r(0)=1-r(1)$, equation (5.4) and $0<r(1) \leq \alpha_{0}$, imply that $0 \leq p(x, 0) \leq 1$. Consider the third equation in (5.3). Then equation (5.5) and $0<r(1) \leq \alpha_{0}$ imply that $0 \leq p\left(x^{\prime}, 0\right) \leq 1$. Therefore the second and third equations yield a consistent value for $p(x, 0)$ and $p\left(x^{\prime}, 0\right)$. Now replace all these quantities in the first equation of $(5.3)$ for $\theta=0,1$.

Somewhat unsurprisingly, Bellman equations cannot provide identifying restrictions for more than one type of agent. The behaviour of one of the groups can therefore be considered as almost arbitrary provided that conditions (5.4) and (5.5) are satisfied. These conditions come from the additional structure imposed by Bayes equations in lemma 5.1. 


\subsection{Identifying restrictions}

We now investigate the identifying power of two popular restrictions. We first assume the additivity of the individual effect in utility

$$
\forall(x, \theta) ; u_{1}^{*}(x, \theta)=u_{1}^{*}(x)+\theta
$$

Second, we impose a weak form of terminal condition: from a certain period onwards, unobserved types do not affect utility levels. For the sake of simplicity, let us consider three periods 0,1 and 2 . Let $x "$ denote observed (by the econometrician) history at time 2 . Then assume that:

$$
\forall\left(x^{\prime \prime}, \theta\right) ; u_{1}^{*}\left(x^{\prime \prime}, \theta\right)=u_{1}^{*}\left(x^{\prime \prime}\right)
$$

Proposition 5.3. Given $G, \beta, r(1)$ and the two previous restrictions (additivity and terminal condition), and provided some rank conditions are satisfied, the utility function $u_{1}^{*}($.$) is just identified at periods 0$ and 1 .

\section{Proof. See Appendix D}

The set of moment conditions given in lemma 5.1 still forms the basis of this result. We have seen in proposition 5.2 that for given $G, \beta$ and $r(1)$, utilities are identified provided we fix two choice probability functions $p\left(x^{\prime}, 1\right)$ and $p(x, 1)$. The degree of underidentification therefore consists in two elements of the set $\mathcal{F}$ of functions mapping the state space into $] 0,1[$. By relating utility functions of the two types, the additivity restriction reduces this degree by one element of $\mathcal{F}$. Excluding heterogeneity at date 2 yields the same reduction in dimensionality. The two parameters $\beta$ and $r(1)$ remain unidentified. They can nevertheless be identified by imposing two exclusion restrictions as in section 4.2.

Finally, the relaxation of assumption (5.2) can be investigated. Unobserved heterogeneity could affect transition probabilities $\operatorname{Pr}\left(x^{\prime} \mid x, d, \theta\right)$ and not preferences if we consider the opposite case to the one just discussed. In this case, the analysis is similar. Begin by expressing the moment conditions and counting the degree of functional underidentification. Then impose identifying restrictions to match the degree of underidentification. For instance, the assumption above 
on terminal dates could be translated into equal transition probabilities for the two groups from a terminal date onwards. The additivity assumption is not more difficult to translate. It consists of a given deterministic relationship that exists between the transition probability functions of the two groups. The identification result follows from this.

\section{Conclusion}

It is well known that dynamic discrete choice models are underidentified. The good news is that the degree of underidentification can be determined and the power of popular identifying restrictions, both with and without correlated fixed effects, can be investigated.

The degree of underidentification consists of the discount factor, the distribution function of random shocks in preferences, and preferences in a reference alternative. We show that, unlike the static case, the differences between utility levels are not identified. In the absence of further information on value function levels, current value functions are the only structural object that can be identified. Imposing a terminal date or assuming the existence of an absorbing state does not carry identifying power, while imposing exclusion or parametric restrictions allows us to identify the discount factor. The degree of underidentification is even larger in the case with unobserved heterogeneity. However, in this case, additivity of individual effects and a weak terminal condition are shown to be powerful identifying restrictions.

The identification strategy based on moments naturally leads to the construction of method-of-moment estimators that can be implemented even with correlated individual effects. We are not advocating the unconditional use of methods of moments to estimate these models, however, moment condition analysis is shown to be a very powerful tool to investigate identification. Given that a model is identified, other methods such as maximum likelihood or iterated method of moments (Aguirregabiria and Mira, 2000) could be used for estimation purposes. The comparison of the statistical properties of maximum likelihood and method of moments, as well as their numerical aspects would be a useful exercise (see Hotz et 
al. [1994]). With its different set of moment conditions, our analysis could help to shed new light on this issue. A Monte Carlo investigation of both methods is clearly needed but left for further research. 


\section{Appendix}

\section{A. Proof of Lemma 3.1}

Start from Bellman equations (2.2). The expected future value function can be written as:

$$
E\left(\max v_{j}\left(x^{\prime}, \tilde{\varepsilon}^{\prime}\right) \mid d=i, x\right)=E\left(E_{G}\left(\max v_{j}\left(x^{\prime}, \tilde{\varepsilon}^{\prime}\right) \mid x^{\prime}\right) \mid d=i, x\right)
$$

where:

$$
\begin{gathered}
E_{G}\left(\max v_{j}\left(x^{\prime}, \tilde{\varepsilon}^{\prime}\right) \mid x^{\prime}\right)=E_{G}\left(v_{K}\left(x^{\prime}, \tilde{\varepsilon}^{\prime}\right) \mid x^{\prime}\right)+E_{G}\left(\max \left(v_{j}\left(x^{\prime}, \tilde{\varepsilon}^{\prime}\right)-v_{K}\left(x^{\prime}, \tilde{\varepsilon}^{\prime}\right)\right) \mid x^{\prime}\right) \\
=v_{K}^{*}\left(x^{\prime}\right)+E_{G}\left(\max \left(v_{j}\left(x^{\prime}\right)-v_{K}\left(x^{\prime}\right)+\tilde{\varepsilon}_{j}-\tilde{\varepsilon}_{K}\right) \mid x^{\prime}\right) \\
=v_{K}^{*}\left(x^{\prime}\right)+R\left(\tilde{w}\left(x^{\prime}\right) ; G\right)
\end{gathered}
$$

by definition of $R(. ; G)$ and where $\widetilde{w}=\left(w_{1}, \ldots, w_{K-1}, 0\right)$, and $w_{j}=v_{j}^{*}-v_{K}^{*}$. This new expression of the continuation value is then plugged back into (2.2). After subtracting $v_{K}^{*}(x)$ from both right and left hand sides, we get, for $i<K$ :

$$
w_{i}(x)=u_{i}^{*}(x)+\beta E\left(R\left(\tilde{w}\left(x^{\prime}\right)\right) \mid x, d=i\right)+\beta E\left(v_{K}^{*}\left(x^{\prime}\right) \mid x, d=i\right)-v_{K}^{*}(x)
$$

and for the reference alternative $K$ :

$$
v_{K}^{*}(x)=u_{K}^{*}(x)+\beta E\left(R\left(\tilde{w}\left(x^{\prime}\right)\right) \mid x, d=K\right)+\beta E\left(v_{K}^{*}\left(x^{\prime}\right) \mid x, d=K\right) .
$$

We replace $v_{K}^{*}(x)$ in (A.1) by (A.2) and we use (3.1) to prove the result.

\section{B. Proof of Proposition 3.2}

The proof is by construction. Fix $c \in C$. As $G$ is fixed, derive functions $q$ and $R$. As $\beta, u_{K}^{*}(X)$ and $v_{K}^{*}\left(X^{\prime}\right)$ are fixed, Bellman equations (3.3) define a unique vector $\left(u_{1}^{*}(X), \ldots, u_{K-1}^{*}(X)\right)$ as a function of the data. Equation (3.2) also defines a unique vector $\left(v_{1}^{*}\left(X^{\prime}\right), \ldots, v_{K-1}^{*}\left(X^{\prime}\right)\right)$. Therefore, for any $c \in C, b=b(c)$ is defined uniquely as a function of $c$ and observationally equivalent to $b^{\prime}=b\left(c^{\prime}\right)$ for any $c^{\prime} \in C$.

\section{Proof of Lemma 5.1}

The only informative Bellman equation concerns alternative 1 . Using $u_{2}^{*}(X, \theta)=0$ and $v_{2}^{*}\left(X^{\prime}, \theta\right)=0$, lemma 3.1 yields:

$$
q(p(x, \theta) ; G)=u_{1}^{*}(x, \theta)+\beta E\left(R\left(q\left(p\left(x^{\prime}, \theta\right) ; G\right) ; G\right) \mid d=1, x\right)
$$


where $p(x, \theta)=\operatorname{Pr}(d=1 \mid x, \theta)$. What the data give us, apart from the transition process $\operatorname{Pr}\left(x^{\prime} \mid x, d=1\right)$ which does not depend on $\theta$ by assumption (5.2), is the choice probability $s(\tilde{x})=p(d=1 \mid \tilde{x})$ which is the mixture:

$$
\forall \tilde{x} \in \tilde{X}, s(\tilde{x})=p(\tilde{x}, 1) \operatorname{Pr}(\theta=1 \mid \tilde{x})+p(\tilde{x}, 0) \operatorname{Pr}(\theta=0 \mid \tilde{x}) .
$$

Finally, assumption (5.2) implies that if $x^{\prime}$ is a subsequent history of $(x, d=1)$ :

$$
\operatorname{Pr}\left(\theta=1 \mid x^{\prime}\right)=\frac{\operatorname{Pr}\left(\theta=1, x^{\prime} \mid x, d=1\right)}{\operatorname{Pr}\left(x^{\prime} \mid x, d=1\right)}=\operatorname{Pr}(\theta=1 \mid x, d=1)
$$

since $x^{\prime} \perp \theta \mid x, d$. Therefore:

$$
\operatorname{Pr}\left(\theta=1 \mid x^{\prime}\right)=p(x, 1) \frac{\operatorname{Pr}(\theta=1 \mid x)}{s(x)}=\frac{p(x, 1) r(1)}{s(x)}
$$

which is the source of dynamic selection biases. The first part of the lemma is given by (C.1) and (C.2). Use (C.3) in (C.2) to prove the last expression.

\section{Proof of Proposition 5.3}

Lemma 5.1 gives the structural restrictions. As there are three dates, there are now two sets of Bellman equations for each type:

$$
\begin{aligned}
& \theta=0,1 ; u_{1}^{*}(x)=q(p(x, \theta))-\theta-\beta E\left(R\left(q\left(p\left(x^{\prime}, \theta\right)\right)\right) \mid x, d=1\right) \\
& \theta=0,1 ; u_{1}^{*}\left(x^{\prime}\right)=q\left(p\left(x^{\prime}, \theta\right)\right)-\theta-\beta E\left(R\left(q\left(p\left(x^{\prime \prime}, \theta\right)\right)\right) \mid x^{\prime}, d=1\right)
\end{aligned}
$$

where additivity of individual effects is used. At the last period, heterogeneity is assumed to be excluded from preferences (terminal date assumption). It implies that decisions may depend on $\theta$ through past decisions $x "$ but not directly:

$$
\forall\left(x^{\prime \prime}, \theta\right) ; p\left(x^{\prime \prime}, \theta\right)=s\left(x^{\prime \prime}\right) .
$$

To eliminate $u_{1}^{*}($.$) , take differences in (D.1) and (D.2) between the two types which$ yields:

$$
\begin{gathered}
q(p(x, 1))-q(p(x, 0))=1+\beta E\left(R\left(q\left(p\left(x^{\prime}, 1\right)\right)\right)-R\left(q\left(p\left(x^{\prime}, 0\right)\right)\right) \mid x, d=1\right) \\
q\left(p\left(x^{\prime}, 1\right)\right)-q\left(p\left(x^{\prime}, 0\right)\right)=1
\end{gathered}
$$

where the last equation is implied by (D.3).

Mixture equations are given by lemma 5.1:

$$
\begin{aligned}
s(x) & =p(x, 1) r(1)+p(x, 0) r(0) \\
s\left(x^{\prime}\right) & =p\left(x^{\prime}, 1\right) \frac{p(x, 1) r(1)}{s(x)}+p\left(x^{\prime}, 0\right) \frac{p(x, 0) r(0)}{s(x)}
\end{aligned}
$$

The population "functions" $s(x), s\left(x^{\prime}\right)$ are obtained from the data. Provided $r(1)$ and $\beta$ are given, the four unknowns $\left\{p(x, 0), p(x, 1), p\left(x^{\prime}, 0\right), p\left(x^{\prime}, 1\right)\right\}$ could be recovered from (D.4)-(D.7) if this system of equations is locally invertible. Invertibility is equivalent to rank conditions that we assume to be satisfied. Once these unknowns are solved for, $u_{1}^{*}($.$) can be recovered from (D.1)-(D.2).$ 


\section{REFERENCES}

Aguirregabiria, V., and P. Mira, 2000, "Swapping the Nested Fixed Point Algorithm: A Class of Estimators for Discrete Markov Decision Models", Working Paper, CEMFI, Madrid.

Altug, S., and R. Miller, 1998, "The Effect of Work Experience on Female Wage and Labour Supply", Review of Economic Studies, 65:45-85.

Belzil, C., and J. Hansen, 1997, "Estimating the Returns to Education from a Non-Stationary Dynamic Programming Model", paper presented at the AEA Conference, Maastricht.

Buchinsky, M., and P. Leslie, 1997, "Educational Attainment and the Changing U.S. Wage Structure", Working Paper, Department of Economics, Yale University.

Cameron, S.V., and J.J. Heckman, 1998, "Life-Cycle Schooling and Dynamic Selection Bias: Models and Evidence for Five Cohorts of American Males", Journal of Political Economy, 106:262-333.

Chamberlain, G., 1984, "Panel Data", in eds Z. Griliches and M. Intriligator, Handbook of Econometrics, North Holland:Amsterdam, 2:1247-1318.

Eckstein, Z., and K. Wolpin, 1989, "The Specification and Estimation of Dynamic Discrete Choice Models", Journal of Human Resources, 24:562-98.

Eckstein, Z., and K. Wolpin, 1999, "Why Youths Drop Out of High School: The Impact of Preferences, Opportunities and Abilities", Econometrica, 67:12951340 .

Hotz, V.J., and R.A. Miller, 1993, "Conditional Choice Probabilities and the Estimation of Dynamic Models", Review of Economic Studies, 60:497-529.

Hotz, V.J., R.A. Miller, S. Sanders and J. Smith, 1994, "A Simulation Estimator for Dynamic Models of Discrete Choice", Review of Economic Studies, 61:265-289.

Keane, M. and K. Wolpin, 1996, "The Career Decisions of Young Men", Journal of Political Economy, 105:473-522.

Koning, R. and G. Ridder, 1996, "The Identification of Discrete Choice Models", Working Paper, Vrije Universiteit Amsterdam.

Manski, C.F., 1988, "Identification of Binary Response Models", Journal of the American Statistical Association, 83:729-38

Manski, C.F., 1992, "Adolescent Econometricians: How do Youth Infer the Returns to Schooling?", in eds C.T. Clotfelter and M. Rotschild, Studies of Supply and Demand in Higher Education, NBER and The Univesity of Chicago Press: Chicago.

Manski, C.F., 1993, "Dynamic Choices in Social Settings", Journal of Econometrics, 58:121-136.

Matzkin, R., 1994, "Restrictions of Economic Theory in Nonparametric Methods", in eds R. Engle and D. McFadden, Handbook of Econometrics, North Holland:Amsterdam, 4: 2524-2554 
Newey, W.K., and, D., McFadden, 1994, "Large Sample Estimation and Hypothesis Testing", in eds R. Engle and D. McFadden, Handbook of Econometrics, North Holland:Amsterdam, IV: 2113-241.

Rust, J., 1994, "Structural Estimation of Markov Decision Processes" in eds R. Engle and D. McFadden, Handbook of Econometrics, North Holland:Amsterdam, IV: 3081-143.

Rust, J., 1996, "Numerical Dynamic Programming in Economics", in eds H.M. Amman, D.A. Kendrick and J. Rust, Handbook of Computational Economics, North Holland: Amsterdam.

Taber, C.R., 2000, "Semiparametric Identification and Heterogeneity in Discrete Choice Dynamic Programming Models", Journal of Econometrics, 96(2):20129 . 